\title{
Universal Flow-Driven Conical Emission in Ultrarelativistic Heavy-Ion Collisions
}

\author{
Barbara Betz, ${ }^{1}$ Jorge Noronha, ${ }^{1}$ Giorgio Torrieri, ${ }^{1,2}$ Miklos Gyulassy, ${ }^{1}$ and Dirk H. Rischke ${ }^{2,3}$ \\ ${ }^{1}$ Department of Physics, Columbia University, New York, 1002\%, USA \\ ${ }^{2}$ Frankfurt Institute for Advanced Studies (FIAS), Frankfurt am Main, Germany \\ ${ }^{3}$ Institut für Theoretische Physik, Johann Wolfgang Goethe-Universität, Frankfurt am Main, Germany
}

\begin{abstract}
The double-peak structure observed in soft-hard hadron correlations is commonly interpreted as a signature for a Mach cone generated by a supersonic jet interacting with the hot and dense medium created in ultrarelativistic heavy-ion collisions. We show that it can also arise due to averaging over many jet events in a transversally expanding background. We find that the jet-induced awayside yield does not depend on the details of the energy-momentum deposition in the plasma, the jet velocity, or the system size. Our claim can be experimentally tested by comparing soft-hard correlations induced by heavy-flavor jets with those generated by light-flavor jets.
\end{abstract}

PACS numbers: 12.38.Mh, 25.75.Bh, 25.75.Gz

RHIC data have convincingly shown [1-4] that a hot and dense medium is created in ultrarelativistic collisions of heavy ions. This medium is most likely the so-called quark-gluon plasma (QGP), predicted by quantum chromodynamics to be the hot and dense phase of strongly interacting matter [5]. The QGP was found to behave like an almost perfect fluid and to be opaque to jets created in the initial stage of the collision [6]. This raises the possibility of using the energy deposited by the jet in the medium, observable by correlations of soft and hard particles, as a probe of the medium's properties.

The experimental soft-hard correlation function $7-10$ exhibits an interesting double-peak structure at angles opposite to the trigger jet. It has been suggested 11, 12 that such a structure is evidence for Mach cones. If the QGP is an opaque low-viscosity fluid, Mach cones result from the interference of sound waves generated by the energy deposited by a supersonic jet [13].

The Mach cone leads to an excess of low- $p_{T}$ hadrons being emitted at an angle that is roughly $\pi-\phi_{M}$ with respect to the trigger jet, where the Mach-cone angle $\phi_{M}$ is given by Mach's law, $\cos \phi_{M}=c_{s} / v_{\text {jet }}$. Here, $c_{s}$ is the speed of sound and $v_{\text {jet }}$ is the jet velocity. If the leading parton of the jet is light, we have $v_{\text {jet }} \simeq 1$, while for a heavy-flavor leading parton, $v_{\text {jet }}<1$. Measuring $\phi_{M}$ and $v_{\text {jet }}$, one could in principle extract $c_{s}^{2}=d p / d e$ and hence the QGP equation of state $(\operatorname{EoS}) p(e)$, where $p$ is the pressure and $e$ the energy density.

Previous calculations $14-23$ have shown that the formation of a double-peak structure on the away-side of soft-hard correlations can be very sensitive to the underlying assumptions about the jet-medium interaction [24]. In the case of a static medium [14, 17, 22], there are two factors that impair the observation of conical correlations, even in a perfect fluid. First, the thermal smearing at the freeze-out surface 25 broadens the away-side peak for low- $p_{T}$ particles [14, 17, 20]. Second, if the momentum deposited by the jet is larger than a certain threshold, a diffusion wake moving in the opposite trigger-jet direction may overwhelm any signal from the Mach cone
17, 22] and leads to a single peak on the away-side. Finally, the above discussion neglects the effects of the expanding medium on the signal. The strong longitudinal and transverse expansion of the QGP will distort the Mach-cone signal [26]. In Ref. [27] it was suggested that the diffusion wake may be reduced by transverse flow while longitudinal expansion should lead to a broadening of the double-peak structure [16].

In this letter we demonstrate that the double-peak structure observed on the away-side of soft-hard correlations could be of different origin. In our calculations two effects conspire to create the observed "conical" signal: the averaging over wakes created by jets in different events and the deflection of the particles emitted from the wakes by the collective transverse flow. The resulting "conical" signal is found to be quite robust against variations of the energy-momentum deposition mechanism and the system size. The observed "cone" angle is also nearly independent of the jet velocity, in contrast to the scenario where the double-peak structure is due to Mach cones. Therefore, even for events where $v_{j e t}<c_{s}$ and proper Mach cones are not formed, the intrinsic nonlinearities of the fluid-dynamical equations give rise, after freeze-out, to conical structures on the away-side that are strikingly similar to those produced by a Mach cone.

We use (3+1)-dimensional ideal fluid dynamics [28] to study the evolution of the QGP,

$$
\partial_{\mu} T^{\mu \nu}=S^{\nu} .
$$

The energy-momentum tensor of the fluid is $T^{\mu \nu}=(e+$ $p) u^{\mu} u^{\nu}-p g^{\mu \nu}$, where $u^{\mu}$ is the four-velocity of the fluid. The conservation equations (10) are closed by using an EoS, in our case that for an ideal gas of gluons, $p=e / 3$. The source term $S^{\nu}$ on the right-hand side of Eq. (11) is the energy and momentum deposited by a jet.

We use a transverse initial energy density profile corresponding to particle creation in central $\mathrm{Au}+\mathrm{Au}$ and $\mathrm{Cu}+\mathrm{Cu}$ collisions according to the Glauber model. The maximum temperature is $T=200 \mathrm{MeV}$ for $\mathrm{Au}+\mathrm{Au}$ and $T=176 \mathrm{MeV}$ for $\mathrm{Cu}+\mathrm{Cu}$ (since we use an ideal gas EoS 
the exact value of the initial temperature for a given system does not play an important role in the analysis). In the longitudinal direction, the system is assumed to be elongated over the whole grid, forming a cylinder. In this way, we maximize the effect of transverse flow since there is no additional dilution from longitudinal motion of the fluid. In our coordinates, the $z$-direction defines the beam axis and the associated jet moves along the $x$-direction.

Assuming that the energy and momentum lost by the jet thermalizes quickly, we use the source term

$$
S^{\nu}(x)=\int_{\tau_{i}}^{\tau_{f}} d \tau \frac{d M^{\nu}}{d \tau} \frac{u_{\alpha} j^{\alpha}}{u_{0, \beta} j_{0}^{\beta}} \delta^{(4)}\left[x-x_{\mathrm{jet}}(\tau)\right],
$$

with the proper-time interval of the jet evolution $\tau_{f}-$ $\tau_{i}$, the (constant) energy and momentum loss rate $d M^{\nu} / d \tau=(d E / d \tau, d \vec{M} / d \tau)$, and the location of the jet $x_{\text {jet }}$. The factor $u_{\alpha} j^{\alpha} / u_{0, \beta} j_{0}^{\beta}$, where $j^{\alpha}$ is the fourcurrent of color charges $\left(u_{0}^{\beta}, j_{0}^{\beta}\right.$ are the initial fourvelocity and four-current of color charges at the center of the system), takes into account that the medium expands and cools, thus reducing the energy-momentum loss rate. In the following, we shall assume that $j^{\alpha} \sim T^{3} u^{\alpha}$. In non-covariant notation, Eq. (2) reads

$$
\begin{aligned}
S^{\nu}(t, \vec{x}) & =\frac{1}{(\sqrt{2 \pi} \sigma)^{3}} \exp \left\{-\frac{\left[\vec{x}-\vec{x}_{\mathrm{jet}}(t)\right]^{2}}{2 \sigma^{2}}\right\} \\
& \times\left(\frac{d E}{d t}, \frac{d M}{d t}, 0,0\right)\left[\frac{T(t, \vec{x})}{T_{\max }}\right]^{3} .
\end{aligned}
$$

In the following we set $\sigma=0.3 \mathrm{fm}$. A temperature cut of $T_{\text {cut }}=130 \mathrm{MeV}$ is applied to ensure that no energymomentum deposition takes place outside the medium. The jet is assumed to move at a constant velocity through the expanding medium [jet deceleration is not expected to lead to significant changes after freeze-out [22]].

In contrast to Ref. [15], we assume that the parton moving through the QGP stops after it has deposited all of its energy. In the following, we consider the jet to be generated by a $5 \mathrm{GeV}$ parton. One can compare with experimental data by assuming that, after fragmentation, the leading hadron carries $\sim 70 \%$ of the parton's energy, corresponding to a trigger- $p_{T}$ of $3.5 \mathrm{GeV}$.

Since the experimental analysis can trigger on the jet direction but not on the space-time location where the jets are formed, one has to consider different jet trajectories pointing along the same direction but originating from different points $(x, y)$ in the transverse plane [29]. We parametrize them as

$$
x=r \cos \phi, \quad y=r \sin \phi,
$$

where $r=5 \mathrm{fm}$ is chosen to account for the fact that the trigger jet originates from a point close to the surface. A more refined treatment [16] will qualitatively lead to the same result. We consider different values for the azimuthal angle ( $\Delta \phi=15$ degrees) with respect to the trigger axis (negative $x$-axis): $\phi=90, \ldots, 165$ degrees, corresponding to jets travelling through the upper half of the transversally expanding medium, $\phi=180$ degrees, corresponding to a jet travelling along the (positive) $x$ axis, and $\phi=195, \ldots, 270$ degrees, corresponding to jets propagating through the lower half of the medium.

In order to compare to experimental data, one has to convert the fluid into particles. In this work, we use the Cooper-Frye (CF) prescription 25] assuming energymomentum and entropy conservation across the so-called freeze-out hypersurface which we take to be a surface at constant time (isochronous freeze-out). This yields the single-inclusive particle spectrum $d N /\left(p_{T} d p_{T} d y d \phi\right)$.

In the experiment, the trajectory of the jet is not known and one has to measure the azimuthal correlation between the hard particles produced by the trigger jet and the soft particles produced by the associated jet traversing the medium. In our calculation, we mimic this soft-hard correlation function by convoluting the singleinclusive particle spectrum obtained from the CF freezeout (which only considers the away-side particles) with a function representing the near-side jet,

$$
f(\phi)=\frac{1}{\sqrt{2 \pi \Delta \phi^{2}}} \exp \left(-\frac{\phi^{2}}{2 \Delta \phi^{2}}\right),
$$

(here $\Delta \phi=0.4$ ), resulting in a two-particle correlation function

$$
C_{2}(\phi)=A f(\phi)+\int_{0}^{2 \pi} d \phi^{\star} \frac{d N}{p_{T} d p_{T} d y d\left(\phi-\phi^{\star}\right)} f\left(\phi^{\star}\right)
$$

where $(A, \Delta \phi)$ are chosen to simulate the near-side correlation. This function is then event-averaged (indicated by $\langle\cdot\rangle)$, background-subtracted, and normalized, leading to the averaged two-particle correlation function

$$
\langle C F(\phi)\rangle=\mathcal{N}\left[\left\langle C_{2}(\phi)\right\rangle-\frac{d N_{\text {back }}}{p_{T} d p_{T} d y d \phi}\right],
$$

where $d N_{\text {back }} /\left(p_{T} d p_{T} d y d \phi\right)$ is the single-inclusive particle spectrum for an event without jets and $\mathcal{N}^{-1}=$ $d N_{\text {back }} /\left(p_{T} d p_{T} d y\right)$.

Figure 1 shows the two-particle correlation function (77) for $p_{T}=2 \mathrm{GeV}$. The jets are assumed to propagate with $v=0.999$, depositing energy-momentum into the medium according to Eq. (3) with $d E / d t=1 \mathrm{GeV} / \mathrm{fm}$ and $d M / d t=1 / v d E / d t$. This case is referred to as "onshell" deposition in the following. We observe a doublepeak structure resembling a Mach-cone signal. The reason is that the contributions from different jet trajectories, shown in the right panel of Fig. 1 1 for jets traversing the upper half of the medium, add up to a peak at an angle $\phi_{w}<180$ degrees (long-dashed blue line in the left 

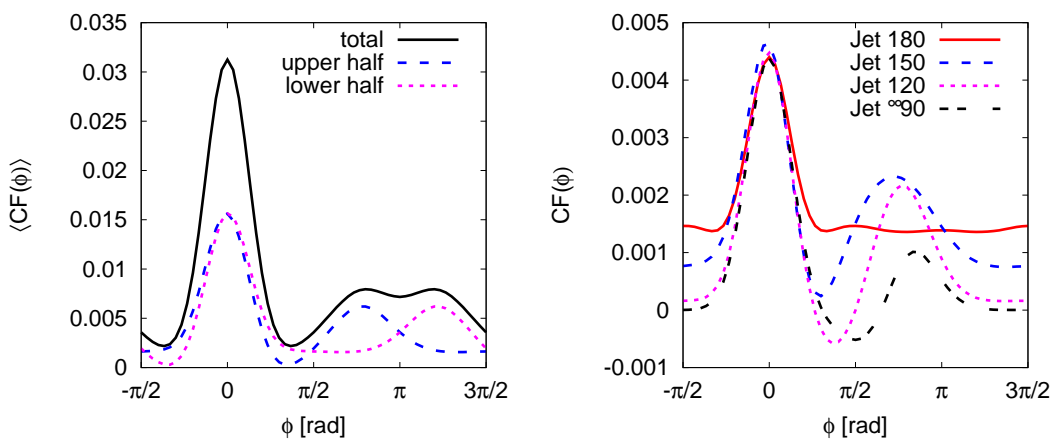

FIG. 1: Left panel: The two-particle correlation function (7) (solid black line) for an associated particle $p_{T}=2$ GeV. The longdashed blue and short-dashed magenta lines represent the averaged contribution from jets traversing only the upper or the lower half of the medium, respectively. Right panel: the unaveraged two-particle correlation function (77) from four representatively chosen different jet trajectories in the upper half of the medium.

panel of Fig. 1), while the contributions from the jets traversing the lower half of the medium produce a peak at an angle $\phi_{w}>180$ degrees (short-dashed magenta line in the left panel of Fig. 11). The value of $\phi_{w}$ depends on how much the transversally expanding medium can deflect the matter in the disturbance caused by the jet. This, in turn, depends on the local flow velocity (both its magnitude and direction with respect to the associated jet) and temperature. The gap between the two peaks on the away-side depends on the $p_{T}$ of associated particles. For small $p_{T}$, thermal smearing is large and the two peaks merge into a single broad away-side peak 30]. The same flow induced peak-to-valley ratio is found within $0.3 \%$ if a coarser $\Delta \phi=30$ degrees initial jet sampling is used. The robustness of the numerical results in Fig. 1 to numerical details underlines the universality of the radial flow mechanism as the primary source of non-Mach away-side azimuthal correlations.

Figure2 shows that a similar double-peak structure as in the on-shell deposition scenario can also be obtained for a pure momentum deposition scenario with $d E / d t=$ 0 , and $d M / d t=1.0 / v \mathrm{GeV} / \mathrm{fm}$. Both scenarios lead to approximately the same apparent "cone" angle. On the other hand, due to thermal smearing a pure energy deposition scenario (with $d E / d t=1 \mathrm{GeV} / \mathrm{fm}$ and $d M / d t=0$ ) only exhibits a single broad peak for $p_{T}=2 \mathrm{GeV}$. For a larger $p_{T}=3 \mathrm{GeV}$, the double-peak structure reappears, with a similar "cone" angle as in the other deposition scenarios. Thus, in an expanding medium, the apparent "cone" shape resulting from averaging over many events is universal to all energy-momentum deposition scenarios, in contrast to the case of a static background 24]. Therefore, we cannot draw conclusions about the jet deposition mechanism, although the pure energy loss scenario seems to be disfavored by the data, since the two peaks appear only at a higher $p_{T}$.

Finally, we demonstrate that the conical emission angle observed in Figs. 1 and 2 also appears for subsonic jets (which should not be the case if it was due to a true Mach cone). We consider a bottom quark with mass $M=4.5 \mathrm{GeV}$, propagating with $v=0.57<c_{s}$ through the medium. The results for the correlation function (7), computed with the on-shell energy-momentum deposition scenario with $d E / d t=1 \mathrm{GeV}$, are shown in Fig. 3. where, for comparison, we also show results for $v=0.75$.

The same double-peak emission structures are found for all jet velocities, even for the subsonic jet, with nearly equal apparent "cone" angles. A comparison between the away-side yield in $\mathrm{Au}+\mathrm{Au}$ and $\mathrm{Cu}+\mathrm{Cu}$ is also shown in Fig. 3. Remarkably, both systems are predicted in this scenario to display a very similar away-side shoulder width in qualitative agreement with recent experimental results from the PHENIX collaboration 31. While the absolute normalization of $\langle C F(\phi)\rangle$ is sensitive to the freeze-out temperature $T_{f}$, we found that the doublepeak structures in $\mathrm{Au}+\mathrm{Au}$ are insensitive to variations of $T_{f}$ between $130 \mathrm{MeV}$ and $160 \mathrm{MeV}$. For $\mathrm{Cu}+\mathrm{Cu}$ the weak dip at $\phi=\pi$ is filled for $p_{T}=2 \mathrm{GeV}$ but reappears at higher $p_{T}$ while the shoulder width remains similar to the $\mathrm{Au}+\mathrm{Au}$ case. Three-particle correlations will be presented elsewhere.

In conclusion, we have shown that a double-peak structure on the away side of soft-hard correlations can originate generally from the coupling of jet fragments to the background transverse collective flow. In addition, the apparent width of the away-side shoulder correlation is "universal" in the sense that it is insensitive to the details of the energy-momentum deposition mechanism as well as to the system size, and that is similar for both supersonic and subsonic "jets". This prediction can be readily tested experimentally by comparing soft-hard correlations induced by heavy-flavor tagged jets [32] with those induced by light-flavor jets at RHIC and LHC.

B.B. is supported by the Alexander von Humboldt foundation via a Feodor Lynen fellowship. M.G., J.N., and B.B. acknowledge support from DOE under Grant 

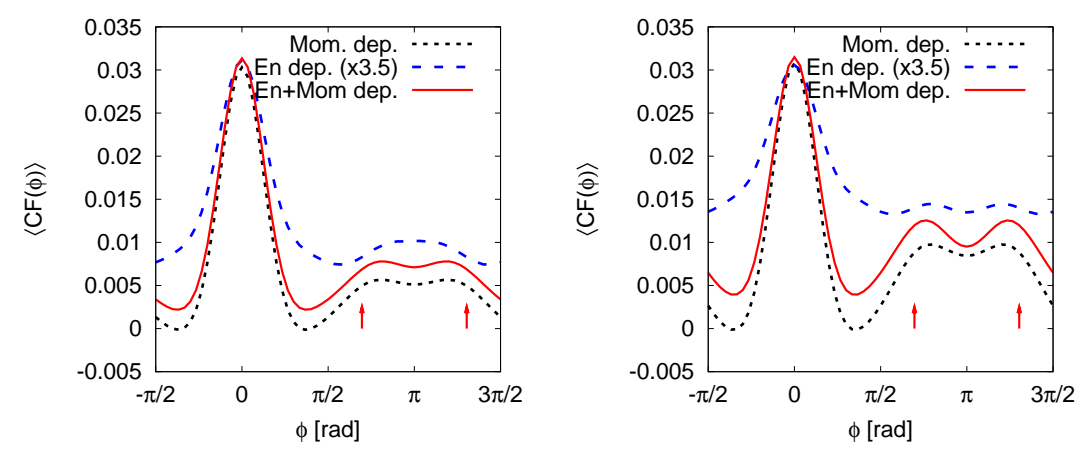

FIG. 2: The two-particle correlation function (7) for jets propagating with $v=0.999$. Solid red line: on-shell energy-momentum deposition. Short-dashed black line: pure momentum deposition. Long-dashed blue line: pure energy deposition (scaled by a factor 3.5). Left panel: $p_{T}=2 \mathrm{GeV}$. Right panel: $p_{T}=3 \mathrm{GeV}$. Arrows indicate the emission angle obtained by Mach's law.

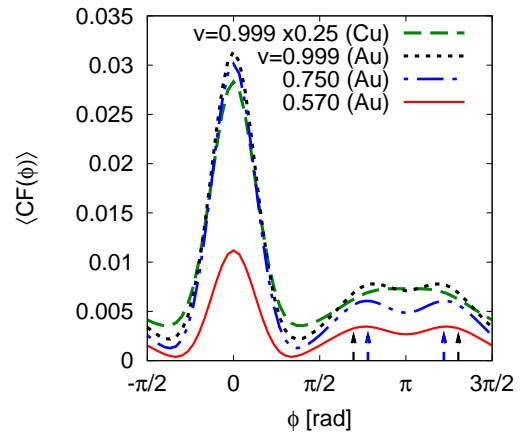

FIG. 3: The two-particle correlation function (7) for jets travelling at $v=0.999$ in central $\mathrm{Cu}+\mathrm{Cu}$ collisions (long-dashed green line) and central $\mathrm{Au}+\mathrm{Au}$ collisions (short-dashed black line), $v=0.75$ (dash-dotted blue line), and $v=0.57$ (solid red line) for $p_{T}=2 \mathrm{GeV}$. For the supersonic jets, the arrows indicate the emission angles obtained by Mach's law.

No. DE-FG02-93ER40764. G.T. acknowledges support from the Helmholtz International Center for FAIR within the framework of the LOEWE program.

[1] I. Arsene et al. [BRAHMS Collaboration], Nucl. Phys. A 757, 1 (2005).

[2] K. Adcox et al. [PHENIX Collaboration], Nucl. Phys. A 757, 184 (2005).

[3] B. B. Back et al., Nucl. Phys. A 757, 28 (2005).

[4] J. Adams et al. [STAR Collaboration], Nucl. Phys. A 757, $102(2005)$.

[5] J. Kapusta, B. Muller and J. Rafelski, "Quark-Gluon Plasma: Theoretical Foundations" (Elsevier, Amsterdam, 2003)

[6] M. Gyulassy and L. McLerran, Nucl. Phys. A 750, 30 (2005); E. V. Shuryak, Nucl. Phys. A 750, 64 (2005).

[7] J. Adams et al. [STAR Collaboration], Phys. Rev. Lett. 95, 152301 (2005).

[8] S. S. Adler et al. [PHENIX Collaboration], Phys. Rev. Lett. 97, 052301 (2006).
[9] A. Adare et al. [PHENIX Collaboration], Phys. Rev. C 78, 014901 (2008).

[10] B. I. Abelev et al. [STAR Collaboration], Phys. Rev. Lett. 102, 052302 (2009).

[11] H. Stoecker, Nucl. Phys. A 750, 121 (2005).

[12] J. Casalderrey-Solana, E. V. Shuryak and D. Teaney, J. Phys. Conf. Ser. 27, 22 (2005) [Nucl. Phys. A 774, 577 (2006)].

[13] L. D. Landau and E. M. Lifshitz, Pergamon Press, New York, (1987).

[14] J. Casalderrey-Solana, E. V. Shuryak and D. Teaney, arXiv:hep-ph/0602183

[15] A. K. Chaudhuri and U. Heinz, Phys. Rev. Lett. 97, 062301 (2006).

[16] T. Renk and J. Ruppert, Phys. Lett. B 646, 19 (2007); Phys. Rev. C 76, 014908 (2007).

[17] B. Betz, M. Gyulassy, D. H. Rischke, H. Stocker and G. Torrieri, J. Phys. G 35, 104106 (2008).

[18] R. B. Neufeld, B. Muller and J. Ruppert, Phys. Rev. C 78, 041901 (2008).

[19] S. S. Gubser, S. S. Pufu and A. Yarom, Phys. Rev. Lett. 100, 012301 (2008).

[20] J. Noronha, M. Gyulassy and G. Torrieri, Phys. Rev. Lett. 102, 102301 (2009).

[21] B. Betz, M. Gyulassy, J. Noronha and G. Torrieri, Phys. Lett. B 675, 340 (2009).

[22] B. Betz, J. Noronha, G. Torrieri, M. Gyulassy, I. Mishustin and D. H. Rischke, Phys. Rev. C 79, 034902 (2009).

[23] W. Li et al., Phys. Rev. C 80, 064913 (2009).

[24] G. Torrieri, B. Betz, J. Noronha and M. Gyulassy, Acta Phys. Polon. B 39, 3281 (2008).

[25] F. Cooper and G. Frye, Phys. Rev. D 10, 186 (1974).

[26] L. M. Satarov, H. Stoecker and I. N. Mishustin, Phys. Lett. B 627, 64 (2005).

[27] R. B. Neufeld, Eur. Phys. J. C 62, 271 (2009).

[28] D. H. Rischke, Y. Pursun, J. A. Maruhn, H. Stoecker and W. Greiner, Heavy Ion Phys. 1, 309 (1995).

[29] A. K. Chaudhuri, Phys. Rev. C 75, 057902 (2007).

[30] B. Betz, J. Noronha, G. Torrieri, M. Gyulassy and D. H. Rischke, Nucl. Phys. A 830, 777C (2009).

[31] A. Adare et al. [PHENIX Collaboration], Phys. Rev. Lett. 98, 232302 (2007).

[32] F. Antinori and E. V. Shuryak, J. Phys. G 31, L19 (2005) 